\title{
AIAA Modeling and Simulation Technologies Conference (MST) Abstract
}

\section{Designing Scenarios for Controller-in-the-Loop Air Traffic Simulations}

Michael Kupfer, Joey Mercer, Chris Cabrall, Jeff Homola, and Todd Callantine Human-Systems Integration Division, San Jose State University/NASA Ames Research Center, Moffett Field, CA USA

\section{Summary}

Within the Human Factors Division at NASA Ames Research Center the Airspace Operations Laboratory $(\mathrm{AOL})$ is developing advanced automation concepts that help to transform the National Airspace System into NextGen, the Next Generation Air Transportation System. High-fidelity human-in-the-loop (HITL) simulations are used as a means to investigate and develop roles, responsibilities, support tools, and requirements for human operators and automation.

This paper describes the traffic scenario design process and strategies as used by AOL researchers. Details are presented on building scenarios for specific simulation objectives using various design strategies. A focus is set on creating scenarios based on recorded real world traffic for terminal-area simulations.

\section{Motivation}

Well prepared traffic scenarios contribute greatly to the success of human-in-the-loop simulations. They translate specific simulation parameters like specific traffic loads or traffic pattern into the situation controllers and pilots will be actually confronted with: lower loads may resemble a current-day scenario, while higher loads may simulate a future timeframe or, many crossing or climbing/descending aircraft will result in a scenario with more conflicts.

Moreover, the realism of the modeled traffic in terms of loads in sectors or on routes, carrier-aircraft type-equipage combinations, flight plans, etc. help the controllers and pilots to trust the simulation environment and, enable them to execute their tasks as close as possible to a real world environment. A thorough vetting process through lab tests with a distributed simulation setup and full scale simulation shakedowns will help to avoid simulation artifacts such as incorrect aircraft ownership, immediate conflicts upon simulation initialization, unrealistic speeds and altitudes or, unrealistic large delay values.

\section{Background}

The AOL has a long history in conducting controller-in-the-loop simulations using the Multi Aircraft Control System (MACS) software. MACS provides an environment for rapid prototyping, HITL air traffic simulations, and evaluation of the current and future air/ground operations.

During previous projects traffic scenarios were developed supporting simulations that focused on a variety of research areas: separation assurance, flexible airspace management, multi-sector flow management, and terminal-area problems. Scenarios that were needed for these simulations provided up to a two and three-fold traffic increase in en-route airspace compared to current-day traffic loads, created particular sector load characteristics that required controllers to apply specific strategies to 
re-route flights out of overloaded areas, and included off-nominal disruptions to terminal-area operations such as go-around events.

Along the efforts of recent simulations, the Traffic Management Advisor for Terminal Metering (TMA-TM), a scheduling automation, the ASTAR-equipped Aircraft Simulator for Traffic Operations Research (ASTOR) simulator, and the option to connect to a FAA-Level-D-certified Boeing 747-400 (B744) simulator have been integrated into the AOL. Using those systems in concert, increased the complexity of the scenario design process and required new scenario building strategies.

\section{Methodology}

Currently real world traffic is being recorded in the NASA Ames Aviation systems division. Those recordings can serve as raw input for a simulation scenario. Visualizing and analyzing the traffic, using the TCSim Route Analyzer/Constructor (TRAC) tool for example, gives insights into the actual traffic pattern, vectoring strategies, flown profiles and speeds, traffic distributions, and traffic and aircraft type mix. TRAC can convert this traffic data into the scenario format used by MACS. Scenario development tools are available inside MACS, and can be used to further modify the raw scenario.

The main scenario design process is defined by the objectives and characteristics of the simulation. Often the first step is to adjust and verify the traffic loads on the different routes by possibly adding, deleting or, moving aircraft. For each aircraft, its route, initialization location, altitude, heading, cruise speed, target altitude, and other parameters have to be defined. Those values are subject to change, however, once aircraft are moved along their routes or in time; for example, to achieve a desired delay distribution at a given point (e.g., meter fix) or arrival sequence. Particular caution is required when developing a scenario to be played out under the influence of winds, where predicted winds may be different from the environment winds.

When using MACS aircraft in conjunction with ASTOR aircraft two separate traffic scenarios need to be built as both systems are being driven by an independent simulation manager expecting files in particular formats. The two scenarios need to interleave seamlessly when running the simulation.

Overall, the scenario design process includes several iterations of modifying the scenario, initially testing it on the developer's local computer, and eventually, testing it in a distributed simulation environment in the actual laboratory where all controller and pilot workstations and other systems are in operation.

\section{Conclusion and Outlook}

The traffic scenario design process takes up a significant portion of an overall simulation preparation. Despite many improvements in the scenario design tools available the process can be very complex. Different scenario designers use different strategies often depending on the simulation objectives and characteristics. With the increased frequency of $\mathrm{AOL}$ simulations and more researchers being involved in the scenario design process an effort is underway to further streamline and improve scenario development. 